\title{
Study on Differential Assist Steering System with Double In-Wheel Motors with Intelligent Controller
}

\author{
Qiang Li, ${ }^{1,2}$ Xiao-li Yu, ${ }^{1}$ Hua Zhang, ${ }^{3}$ and Rui Huang' \\ ${ }^{1}$ Power Machinery \& Vehicular Engineering Institute, Zhejiang University, Hangzhou 310027, China \\ ${ }^{2}$ School of Mechanical \& Automotive Engineering, Zhejiang University of Science and Technology, Hangzhou 310023, China \\ ${ }^{3}$ Zotye Holding Group Co., Ltd., Hangzhou 310052, China
}

Correspondence should be addressed to Qiang Li; liqiang1353@163.com

Received 11 June 2015; Revised 25 November 2015; Accepted 6 December 2015

Academic Editor: Francesco Braghin

Copyright (C) 2015 Qiang Li et al. This is an open access article distributed under the Creative Commons Attribution License, which permits unrestricted use, distribution, and reproduction in any medium, provided the original work is properly cited.

\begin{abstract}
The purpose of this paper is to present a novel controller of differential assist steering system. Firstly, the integrated controller module in electric vehicle is investigated, in which the front axle is refitted for two in-wheel motors. Secondly, genetic algorithm is used to optimize the control decision-making rules of fuzzy controller. Thereafter, the built model is embedded in veDYNA development environment with an offline simulation test of driver/vehicle/road closed-loop system. At last, control strategy, which has been compiled and downloaded to Mirco AutoBox controller prototype, is used for the real vehicle experimental analysis and validation of the proposed controller. Both simulation and experimental results demonstrate that the controller can improve the yaw motion and reduce the slip angle of vehicle. Here, the vehicle is equipped with the in-wheel motors on both sides. At the same time, the controller cannot only track the steering torque indication of driving current, but also preferably maintain the steering assist handle. Furthermore, it can bring about a good road feeling to the driver because of the reduced torque fluctuation.
\end{abstract}

\section{Introduction}

By virtue of the planetary system or gear reducer (such as decelerating structure or torque-increasing system) with direct or indirect mechanical connection, electric driving power can be transmitted from motor to wheel/rim with higher efficiency than the traditional engine power pattern. Adopting a special and simplified structure, the chassis control system, such as TCS, ABS, and ESP, can distribute and actively control the different drive force and brake force acting on every electric vehicle in-wheel motor [1-3]. This direct control method is superior to the traditional electronic control one on account of high real time and independence. Differential assist steering system is suitable for electric vehicle with in-wheel motors, which can in real time adjust the output torque of both left and right side of each in-wheel motor, which produces the driving force between friction road and tire.
The advanced control technology that needs to distribute and control speed and torque at the same time has become a study focus in many universities and research institutes because of numerous uncertainties and nonlinear factors. Therefore, various integrated electromechanical structures with an electric motor on wheel hub and many torque distribution methods have been proposed in $[4,5]$ in order to overcome the drawbacks of traditional power driving patterns and electronic controlling strategies in the dynamic control system of electric vehicle. In [4], an innovative electromechanical device has been presented, which has integrated a brushless DC (BLDC) hub motor with a speedchanging wheel hub. Moreover, the new model of an electric differential system has been discussed. The model is based on the measured performance with one induction motor for each wheel drive. Additionally, several front steering control laws have been designed in [6-8] in order to generate stable yaw torques to improve the handling dynamics and stability. 


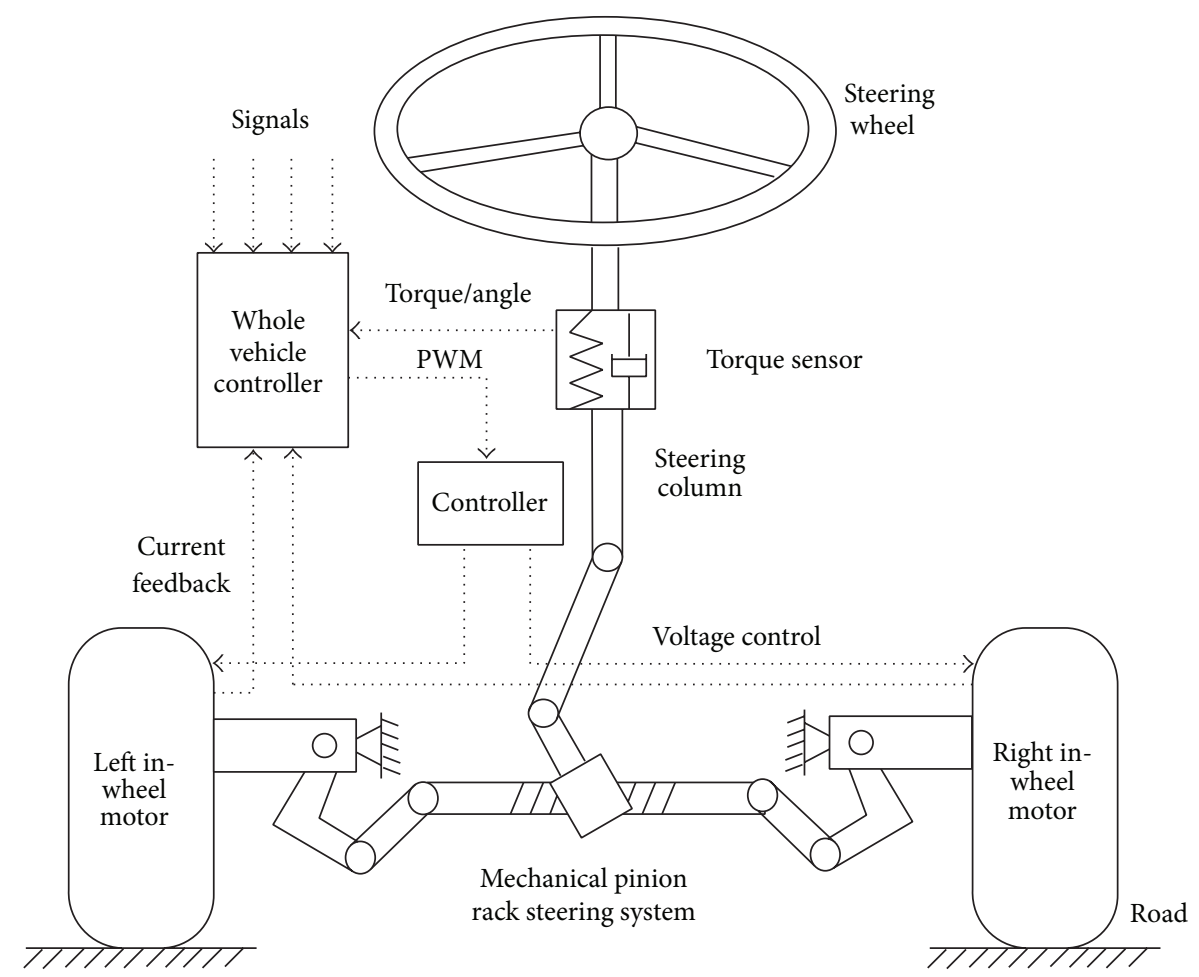

FIGURE 1: Configuration of differential assist steering system of electric vehicle.

In [6], nonlinear optimization forces are integrated with the active front wheel steering and direct yaw moment. And the forces act on the four wheels with torque distribution exerted by the tires. The global optimization of chassis control is carried out [7] by determining the optimal forces based on the actuators of both active rear steering and differential. In [8], a PI feedback control on differential assist steering is used depending on the direct yaw torque and drive toque; however, the precise process control needs to be measured and fitted by plenty of testing data.

This paper tries to design a novel electric controller module for differential assist steering system by combining fuzzy control strategy with genetic algorithm method. In this case, it can achieve the different exported driving torques from front left and right sides of double in-wheel motors on two steering knuckles. The input variables of deviation value are introduced for dynamic performance of fuzzy controller, including the change rate. The output variables of difference values for total driving torque are addressed too. The table of fuzzy control rules is synthesized with optimized GA to generate fuzzy rules for the most important goal as few as possible. The model of whole electric vehicle is presented, which is equipped with double in-wheel motors in veDYNA software. The model is simulated in step response condition to compare with other different control strategies. Finally, the experimental results of both low speed steering condition and double-lane change condition are illustrated to verify the effectiveness and accuracy of electric controller unit.

\section{Differential Assist Steering}

When traditional vehicles that acquire power from burning fuels in internal-combustion engines move on a good smooth road, the steering torque of right side is basically outputted the same as the left side. The steering torques of both sides are only provided by the longitudinal friction forces around the different kingpins. When one side of steering wheels could be electric driving wheel, the process of power transmission path does not need to adopt the function of differential transmission considering the allocation of different torques for mechanical adjustment of the rotational speed. Notably, the torque distribution difference lies in the steering assist torques, which are additionally generated from the in-wheel motors on both sides. The torque difference is equivalent to the driving force variance between left and right sides around the corresponding kingpin. Differential assist and electronic power steering systems are very similar in many aspects. Both need some torque sensors (usually including the angle sensor) to measure the driver input steering torque and steering angle, like the traditional mechanical steering system, such as rack-and-pinion steering, recirculating balllever, and peg steering gear. Figure 1 shows the specific structure and working principle of differential assist steering system, which comprises two in-wheel motors and electric control unit (ECU). The PWM value of driving voltage can be calculated in ECU as desired to achieve the relative relationship of real-time signals information, reflecting various conditions, such as braking fluid pressure, vehicle speed, and CAN bus. The driving module of motor combines 
them with sophisticated control algorithms, which generates suitable terminal voltages for in-wheel motors, including the input of various signals. Those make it possible for right and left in-wheel motors to obtain the appropriate driving torque separately and pair difference according to a realtime regulating PWM due to the current regulation. With regard to electric vehicle, it has the driving power originated from the two front in-wheel motors, which can produce two different driving torques independently. Generally, the steering torque consists of driver's hand steering torque based on the steering gear mechanism and power assist torque caused by the difference of driving torques.

\section{Fuzzy Control Based on Genetic Algorithm}

3.1. Fuzzy Controller. The control technology of differential assist steering system is similar to electric power steering. The control algorithms should be more adaptive in order to deal with the ever-shifting traffic condition, including assist characteristic curves and control laws. It means that the control rules which have basic similarities to human being thinking should be carried out by fuzzy logic process [9]. For example, the driver's input steering torque should be very easy to be described by fuzzy terms such as "heavy," "medium," or "light" that could be transformed and quantized by fuzzy logic sets. A genetic algorithm has been found, and it could be used widely in fuzzy logic, which has one of the active areas named fuzzy controllers. The genetic algorithm is introduced to optimize various membership functions as well as fuzzy rules. The hierarchic control system consists of fuzzy logic, and the genetic algorithm, which allows a discrete and particular description to have continuous and robust interactions with performance, can effectively reduce the complexity of whole system by virtue of adaptive procedure.

3.2. Input/Output Variables of Fuzzy Controller. The ideal yaw rate of the electric vehicle equipped with two in-wheel motors under the steady static condition is expressed as follows:

$$
\omega_{r d}=\frac{u}{L \cdot\left(1+K u^{2}\right)} \cdot \tan \left(\delta_{w}\right),
$$

where $u$ is the longitudinal direction speed, $L$ is the distance from front steering knuckle spindle to rear wheel axle, and $\delta_{w}$ is the front steering angle input by the driver. $K$ is defined as

$$
K=\left(\frac{a}{k_{2}}-\frac{b}{k_{1}}\right) \cdot \frac{m}{L^{2}},
$$

where $a$ and $b$ are the distance from front steering knuckle spindle to the body center of gravity and to the rear wheel axle, respectively. $m$ is vehicle mass; $k_{1}$ and $k_{2}$ are the stiffness of front and rear wheel, separately.

Moreover, the relation between ideal yaw rate and maximum longitudinal adhesion coefficient must satisfy the following formula in any case:

$$
\left|a_{y}\right|=\left|\frac{u^{2}}{R}\right|=\left|\omega_{r_{-}} \cdot u\right| \leq \mu_{\max } \cdot g,
$$

where the lateral acceleration is defined as $a_{y}$, steering turning radius of vehicle trajectory as $R$, the maximum longitudinal adhesion coefficient as $\mu_{\max }$, and the local acceleration of gravity as $g$.

The fuzzy sets are the most important parts of fuzzy logic strategy. The difference of fuzzy sets between actual measurement value and ideal design value is regarded as a generalization set in that memberships are totally graded between 0 and 1 . The signal fusions of normalized difference with yaw rate and side-slip angle are produced by fuzzy sets. The deviation model of dynamic vehicle equations with dynamic response is represented by

$$
\begin{aligned}
e & =\lambda \cdot\left(\omega_{r_{-}}-\omega_{r_{-} a}\right)+(1-\lambda) \cdot\left(\beta_{-d}-\beta_{-a}\right) \\
& =\lambda \cdot e+(1-\lambda) \cdot e_{c},
\end{aligned}
$$

where $\lambda$ is the weight coefficient and $\omega_{r_{-} a}$ and $\beta_{-a}$ are actual yaw rate and side-slip angle of measurable parameters, respectively. $\beta_{-d}$ is expected side-slip angle. In general, the expected vehicle side-slip angle is set to be zero. Two input variables of fuzzy controller are the deviation value of dynamic performance $e$ and deviation change rate $e_{c}$. The output variable $\Delta M_{\mathrm{lr}}$ is a difference between values of driving torque from in-wheel motor concerning left and right side.

3.3. Input/Output Variables of Fuzzy Controller. Without loss of generality, given a domain of discourse, the fuzzy set of two input values can be described with a set of seven linguistic variables such as NB (negative big), NM (negative medium), NS (negative small), ZE (zero), PS (positive small), PM (positive medium), and $\mathrm{PB}$ (positive big). The shapes of the membership functions for two input variables ( $e$ and $e_{c}$ ) are ladder functions. The triangle functions are modified separately. The membership function of deviation $e$ is indicated in Figure 2.

In order to cover and meet as many different driving conditions as possible, the fuzzy set of output values can be described by a set of nine linguistic variables except the abovementioned, including NVB (negative very big) and PVB (positive very big). It is shown in Figure 3.

Given domains of a set of input and output variables, the fuzzy rules can be written on the basis of linear parameter spaces. The Takagi-Sugeno model is shown directly as follows:

Rule i:

$$
\text { IF } e \text { is } \mathbf{E}_{j} \text { and } e_{c} \text { is } \mathbf{E}_{c k} \text { THEN turn } \Delta M_{\mathrm{lr}} \text { to } S_{j k} \text {, }
$$

where $i=1,2,3, \ldots, 49 ; j, k=1,2,3, \ldots, 7$.

As shown in Table 1, the fuzzy rule-base control system of decision-making can draw actually two input variables to output variables by using fuzzification, fuzzy inference, and defuzzification methods.

\section{Decision-Making Fuzzy Control of GA (Genetic Algorithm) Coding}

The table of decision-making fuzzy control can be represented by string codes of chromosome using 12-dimensional 
TABLE 1: Decision-making fuzzy control.

\begin{tabular}{lccccccc}
\hline $\begin{array}{l}\text { Output } \\
\text { variable }\end{array}$ & NB & NM & NS & ZE & PS & PM & PB \\
\hline $\begin{array}{l}\text { Deviation } \\
\text { change rate }\end{array}$ & & & & & & & \\
NB & NVB & NVB & NB & NM & NB & NS & ZE \\
NM & NVB & NVB & NM & NS & NS & ZE & PS \\
NS & NB & NB & ZE & NS & ZE & PS & PM \\
ZE & NB & NM & PS & ZE & NS & PM & PB \\
PS & NM & NS & ZE & PS & ZE & PB & PVB \\
PM & NS & ZE & PS & PM & PB & PVB & PVB \\
PB & ZE & PS & PM & PB & PVB & PVB & PVB \\
\hline
\end{tabular}

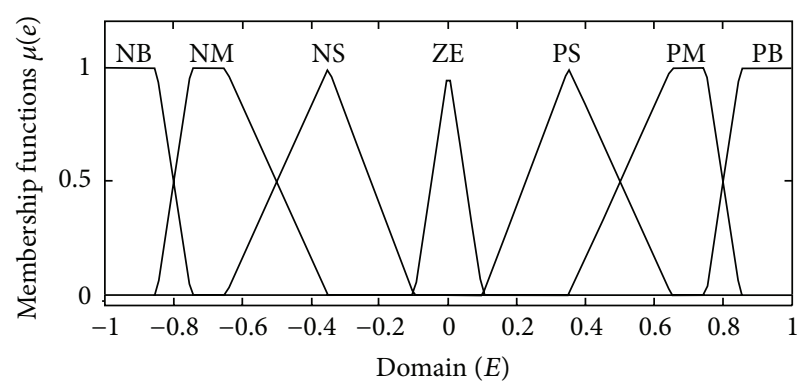

FIgURE 2: Membership functions of deviation.

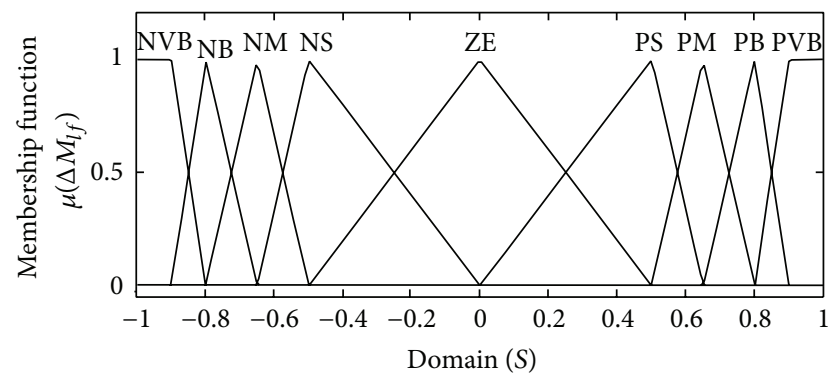

FIGURE 3: Membership functions of output variable.

binary, such as 10100100001 1. Bit 0 (the last digit) is designed as optimization bit to determine whether this rule needs to be changed or adjusted. If any of the other string codes is modified including the first bit to the tenth bit, this bit is set to be 1 , otherwise 0 . Bits 1-4 are represented as output value $\Delta M_{\mathrm{lr}}$. Bits 5-7 and Bits 8-10 are expressed as two input values $\left(e\right.$ and $e_{c}$ ). Bit 11 (the first digit) is used as a flag bit in order to judge whether this rule is adopted. The value of flag bit only has 0 or 1 , which signifies each condition rejection or acceptation.

For example, the string code of a control rule is 101001000011 , which means that $e$ and $e_{c}$ are NM and $\Delta M_{\mathrm{lr}}$ is NB. It is evident that this rule is adopted after optimization. Another example, the string code of a control rule is 110010100110 , which means that $e$ is PS and $e_{c}$ is ZE and $\Delta M_{\mathrm{lr}}$ is NS. Similarly, this rule is adopted without optimization.

Seed selection method of GA can guarantee global convergence relationship [10]. The optimal individual is chosen to enter the next generation of evolutionary population. The optimization process can be specifically solved by five steps as follows.

(1) Fitness. All of the individual fitness values of population $N(0)$ are calculated. In order to estimate the performance of the controller, the objective function in discrete form is designed as follows:

$$
J(x)=\sum_{k=1}^{t_{s}} \omega_{1} \cdot|e(k)|+\omega_{2} \cdot\left|e_{c}(k)\right|+\omega_{3} \cdot\left|\Delta M_{\mathrm{lr}}(k)\right|,
$$

where $t_{s}$ is regarded as the effective duration of controller and $\omega_{1}, \omega_{2}$, and $\omega_{3}$ are different weight coefficients of three different polynomials, respectively.

The fitness function is given as follows:

$$
f(x)=\frac{1}{1+c_{4} \cdot J(x)} \cdot c_{5},
$$

where $C_{4}$ is the control parameter of function sensitivity and $C_{5}$ is the enlargement factor. Because the objective function is small enough, the enlargement factor is added to avoid a large number of floating point operations.

(2) Choice. According to the value of fitness degree in current generation population, those relatively excellent individual populations that will get into next round of the evolutionary are replicated.

(3) Crossover. As for the meaning and scope of different control rules, it is easy to produce the comparatively sick and reasonable individual. Only difference absolute value of fitness degree less than 1.6 was allowed to go on next crossover in order to avoid the occurrence of sudden gene variation.

(4) Mutation. The simulation annealing algorithm which has a great ability of local search can effectively bring out the global optimum. One of the parent individuals can be described as $x=\left(x_{1}, x_{2}, \ldots, x_{k}, \ldots, x_{n}\right) \cdot x^{\prime}=$ $\left(x_{1}, x_{2}, \ldots, x_{k}^{\prime}, \ldots, x_{n}\right)$ can be expressed as a new individual after mutation. The variation of mutant gene ranged from $a_{k}$ to $b_{k}$ can be calculated as follows:

$$
x_{k}^{\prime}=x_{k}-a_{k}+\Delta(n, y),
$$

where $\Delta(n, y)$ is defined as

$$
\Delta(n, y)=\left|b_{k}-x_{k}\right|\left(1-r^{T^{\lambda}}\right),
$$

where $r$ is chosen randomly between 0 and $1, \lambda$ determined by the evolution algebra is dependent on the degree of variation of parameters, and $T$ is the temperature of simulation annealing process that can be given as follows:

$$
T=1-\frac{f}{f_{\max }},
$$




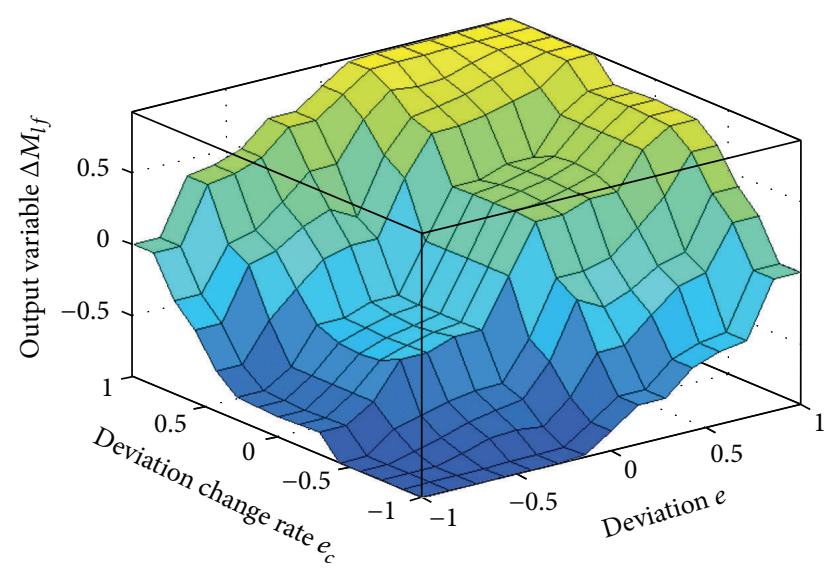

FIGURE 4: Surface of fuzzy control rules optimized by genetic algorithm.

where $f$ and $f_{\max }$ are the fitness degree of given population and the maximum value of current certain generation.

(5) Repeat (3) and (4). The optimization process will be accomplished until the evolution algebra is satisfied to the set value. It is the important factor of crossover probability and mutation probability influencing the performance of genetic algorithm. The maximum fitness degree of each current generation is larger than the previous generation using the seed law. Through the optimization process of genetic algorithm, four fuzzy rules were abandoned due to redundancy and twenty-four rules were optimized. After the optimization of fuzzy genetic algorithm is finished, the surface of fuzzy control rules is shown in Figure 4.

\section{Results and Discussions}

5.1. Simulation Analysis. The simulation of parallel hybrid electric vehicle by using vehicle dynamics software TESIS veDYNA is built based on MATLAB/Simulink platform and then switched from driving power to pure electric mode without connection with engine. The control strategies of output steering torque are implemented on front left and right wheels through optimization GA fuzzy control and differential assist steering is proposed. The specific control structure of simulation strategy is shown in Figure 5.

The simulation environment is installed with many new parameters as follows. The power transfer structure is a full parallel hybrid model. The whole vehicle mass is $960 \mathrm{~kg}$. The front wheel radius is $0.3 \mathrm{~m}$ and wheelbase is $2.10 \mathrm{~m}$. The longitudinal adhesion coefficient is set to be 0.7 . The user's program of electric vehicle is permitted to accelerate from static condition to $80 \mathrm{~km} / \mathrm{h}$. The steady state turning radius is $5.56 \mathrm{~m}$. The longitudinal acceleration minimum value by characterization of vehicle handling stability is $2 \mathrm{~m} / \mathrm{s}^{2}$. The lateral acceleration is allowed to be modified in the range of $0-4 \mathrm{~m} / \mathrm{s}^{2}$. When the model of whole electric vehicle is simulated in step response, various experiments of dynamic simulation were carried out and the yaw rate response of the simulation model with different control strategies was analyzed in Figure 6.

The dynamic response performance of riding was improved more comfortably and yaw stability was ensured through GA optimization of fuzzy controller, whose yaw rate became smoother than without GA in step response process.

5.2. Experimental Validation. Due to the verification of GA optimization of fuzzy controller, the test vehicle equipped with VBOX data acquisition system from inertia sensor of British Racelogic Company is modified on two in-wheel electric motors to original two wheels and tires. Every front wheel load is $540 \mathrm{~kg}$. The capacity of battery that consists of eight pieces is (main parameters including current and voltage) $120 \mathrm{Ah} 96 \mathrm{~V}$. Each electric wheel power is $2.2 \mathrm{~kW}$. Also the steering wheel integrated with sensors from SOHGOH Company of Japan measures the value of steering force and steering angular velocity, which are mounted in steering column instead of original steering wheel. The Mirco AutoBox from dSPACE Company of Germany is fixed in codriver's chair as a rapid prototyping controller.

The road tests under low speed steering with constant radius $20 \mathrm{~m}$ condition and double-lane change condition are accomplished in a round square in order to validate the steering stability performance of differential assist steering system. The test data including vehicle speed, steering torque, steering angle, side-slip angle, and yaw rate and other handling and stability information are recorded for the purpose of analysis on merits and drawbacks of controller by using VBOX realtime acquisition. The currents in every in-wheel motor are measured using LEM current sensors and transferred to realtime monitor in ControlDesk software adopting DS2004 A/D device. The fuzzy controller without GA optimization was compiled and also downloaded to Mirco AutoBox. The steering wheel angle was shown in Figure 7.

Figure 8 presents the comparison of current curves changing between left and right front in-wheel motors with the steering wheel angle range of about 12 degrees. The experimental results show that the hand steering torque input by driver at low vehicle speed is obviously reduced; the deviations of steering torque are within the range of $\pm 0.5 \mathrm{~N} \cdot \mathrm{m}$. The currents of in-wheel motors on both sides can be evaluated to track the driver input hand torque as well as the steering wheel from the intermediate position to the right limit position. By analyzing the current curves shown in Figure 8, it can be observed that the driving current value of left front in-wheel motor becomes larger and the driving current value of right in-wheel motor becomes smaller. The controller of differential assist steering provides better steering feel from driver's subjective evaluation than before.

In the double-lane change test, the electric vehicle speed was held on $40 \mathrm{~km} / \mathrm{h}$. The steering angle, real-time data recording currents of two in-wheel motors with different controllers, the side-slip angle, and yaw rate of test vehicle are shown in Figures 9-12.

It can be found that the driving current value of left inwheel motor with fuzzy controller optimized by the proposed 


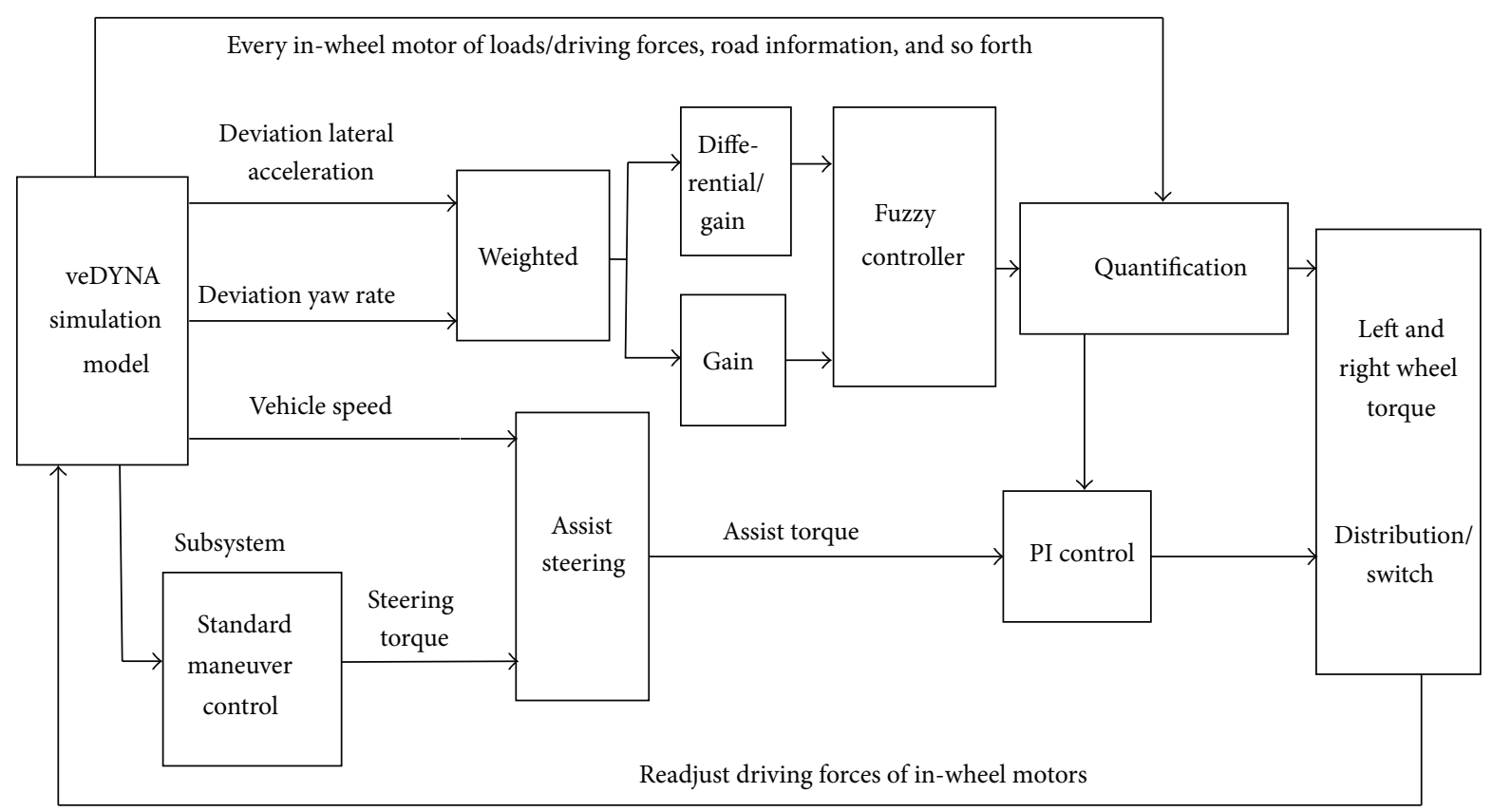

FIGURE 5: Control strategy of differential assist steering.

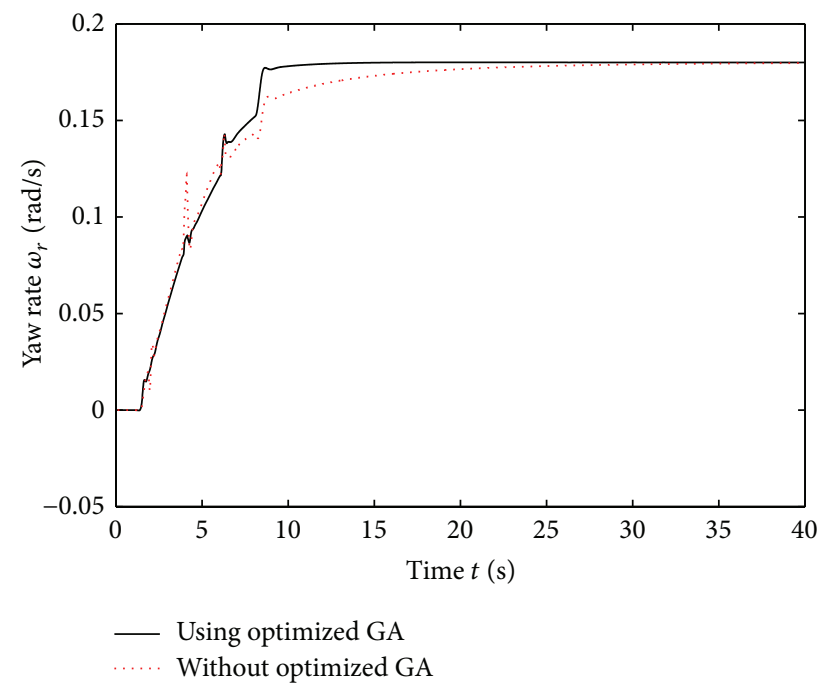

Figure 6: Comparison of yaw rate responses by using different control methods.

GA becomes larger than only with fuzzy controller when the driver's input hand torque ranges from $-3 \mathrm{~N} \cdot \mathrm{m}$ to $3 \mathrm{~N} \cdot \mathrm{m}$. We can find that the new controller can decrease the steering wheel torque fluctuations and provide adequate road sense information for the driver. The characteristics of differential assist steering system are similar to EPS under the condition of keeping total driving force so that the currents of in-wheel motors are suitable to operate with driver's torque via steering wheel.

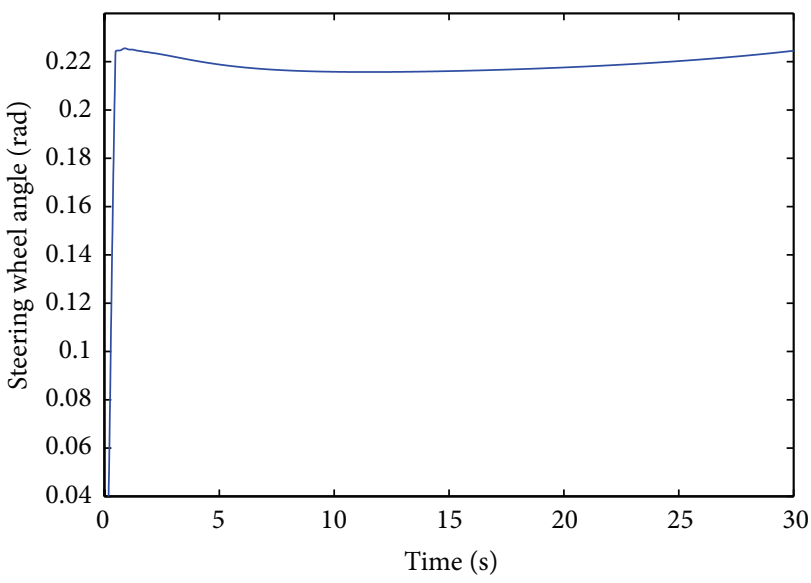

Figure 7: Steering wheel angle with the test driver inputted.

\section{Conclusions}

The fuzzy controller's effectiveness of simulation and experiment of electric vehicle on differential assist steering system for in-wheel motors on both sides is mostly affected. One of the most important reasons is the quality and complexity of the table of decision-making for fuzzy control model after optimization, which are the number of fuzzy rules and two degrees of yaw rate and lateral acceleration of the whole vehicle model. The simple model can be easy to be described by means of tools and modules available in Matlab/Simulink software, which does not substantially blemish possessing the characteristic of the simulation results. Based on the 


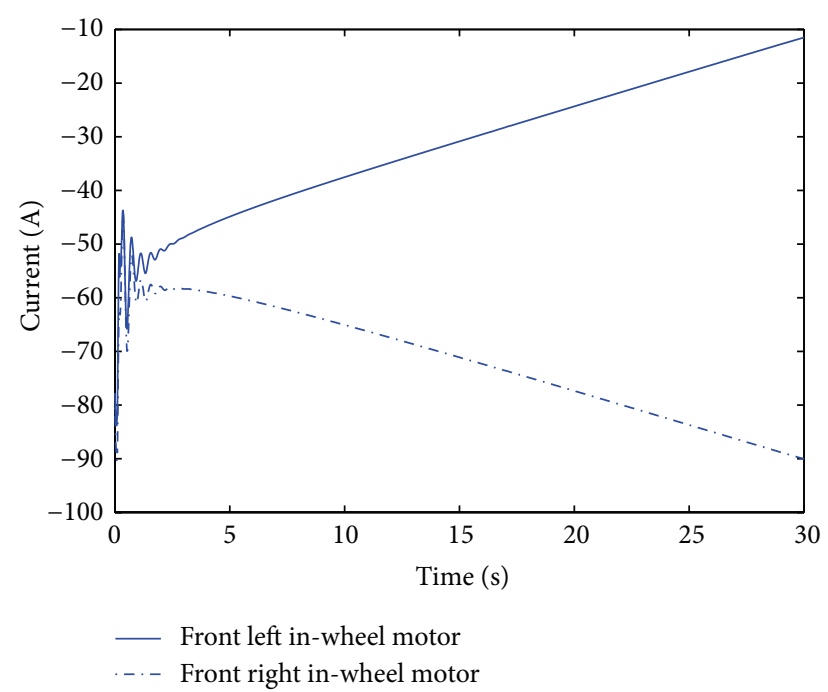

FIGURE 8: Currents of left and right front motor wheels with constant radius.

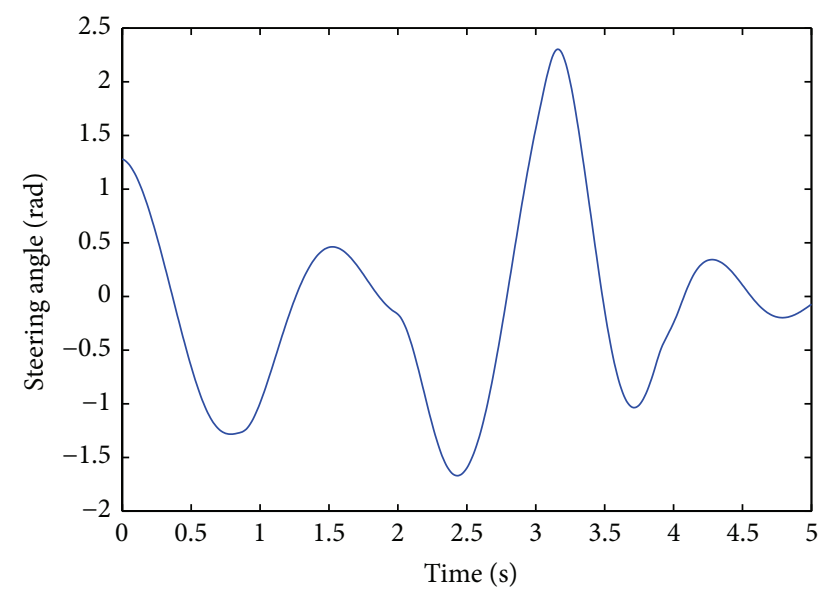

FIGURE 9: Steering wheel angle of test vehicle.

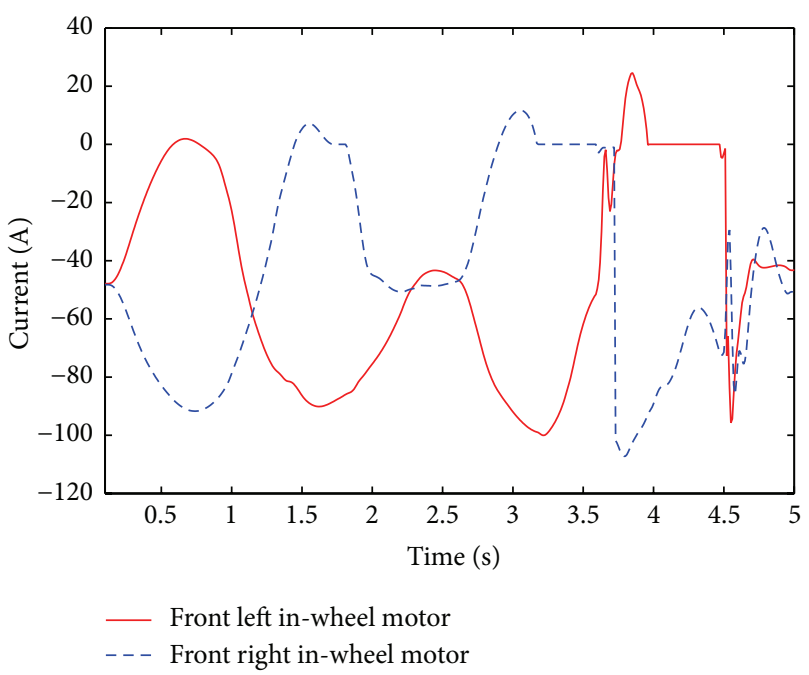

FIGURE 10: Currents of front left and right motor wheels with GA optimized controller.

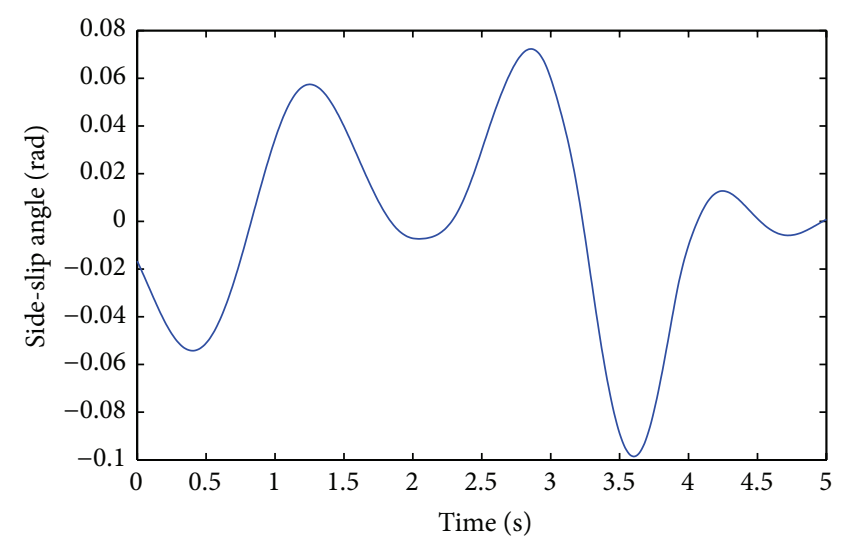

FIGURE 11: Side-slip angle of test vehicle with GA optimized controller.

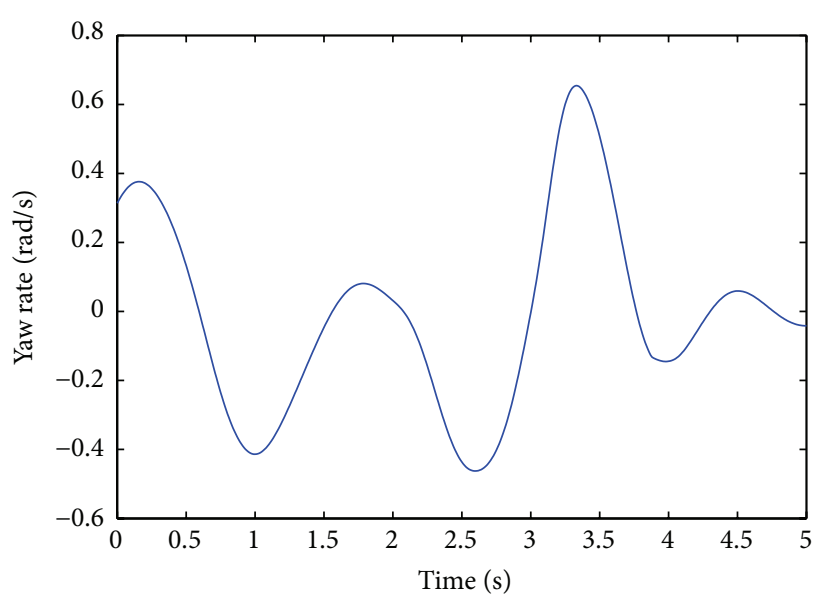

Figure 12: Yaw rate of test vehicle with GA optimized controller.

comparison of the simulation and experimental results for adjusting currents of double in-wheel motors on both sides with the automotive electric control unit combining with different control algorithms, it demonstrates that the fuzzy controller with optimized GA is adequate for engineering practice, such as low speed steering and double-lane change.

\section{Conflict of Interests}

The authors declare that there is no conflict of interests regarding the publication of this paper.

\section{Acknowledgments}

The authors are grateful to the National Natural Science Foundation of China (Grant no. 51476143), Zhejiang Provincial Natural Science Foundation (no. LY13E050023), and Zhejiang Provincial Public Projects (no. 2015C32075) for their supports. 


\section{References}

[1] B. Gasbaoui and A. Nasri, "A novel 4WD electric vehicle control strategy based on direct torque control space vector modulation technique," Intelligent Control and Automation, vol. 3, no. 3, pp. 236-242, 2012.

[2] R. Marino and S. Scalzi, "Integrated active front steering and semiactive rear differential control in rear wheel drive vehicles," in Proceedings of the 17th IEEE IFAC World Congress, pp. 1073210737, Seoul, South Korea, July 2008.

[3] J. S. Lee, Y. J. Ryoo, Y. C. Lim et al., "A neural network model of electric differential system for electric vehicle," in Proceedings of the 26th Annual Conference of the IEEE Industrial Electronics Society, vol. 1, pp. 83-88, October 2000.

[4] Y.-C. Wu and Z.-H. Sun, "Design and analysis of a novel speed-changing wheel hub with an integrated electric motor for electric bicycles," Mathematical Problems in Engineering, vol. 2013, Article ID 369504, 8 pages, 2013.

[5] B. A. Guvenc, T. Acarman, and L. Guvenc, "Coordination of steering and individual wheel braking actuated vehicle yaw stability control," in Proceedings of the IEEE Intelligent Vehicles Symposium (IV'03), pp. 288-293, Columbus, Ohio, USA, June 2003.

[6] C. Zhao, W. Xiang, and P. Richardson, "Vehicle lateral control and yaw stability control through differential braking," in Proceedings of the International Symposium on Industrial Electronics (ISIE '06), pp. 384-389, Montreal, Canada, July 2006.

[7] K. Jalali, T. Uchida, J. McPhee, and S. Lambert, "Development of an advanced fuzzy active steering controller and a novel method to tune the fuzzy controller," SAE International Journal of Passenger Cars-Electronic and Electrical Systems, vol. 6, no. 1, pp. 241-254, 2013.

[8] A. Watts, A. Vallance, A. Fraser et al., "Integrating in-wheel motors into vehicles-real-world experiences," SAE International Journal of Alternative Powertrains, vol. 1, no. 1, pp. 289307, 2012.

[9] P. Cross and X. D. Ma, "Model-based and fuzzy logic approaches to condition monitoring of operational wind turbines," International Journal of Automation and Computing, vol. 12, no. 1, pp. 25-34, 2015.

[10] M. J. Zhang, J. X. Zheng, and J. Zhang, "Selection method of multi-objective problems using genetic algorithm in motion plan of AUV," Journal of Marine Science and Application, vol. 1, no. 1, pp. 81-86, 2002. 


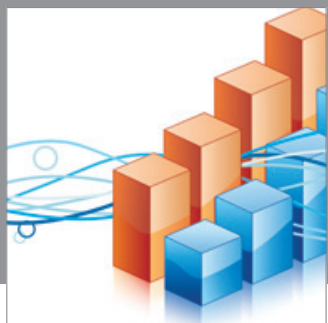

Advances in

Operations Research

mansans

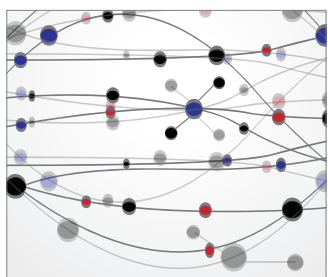

The Scientific World Journal
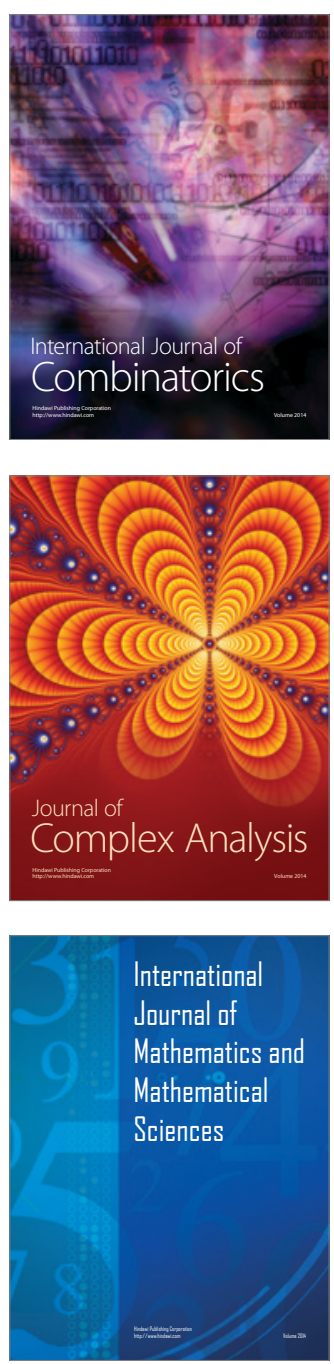
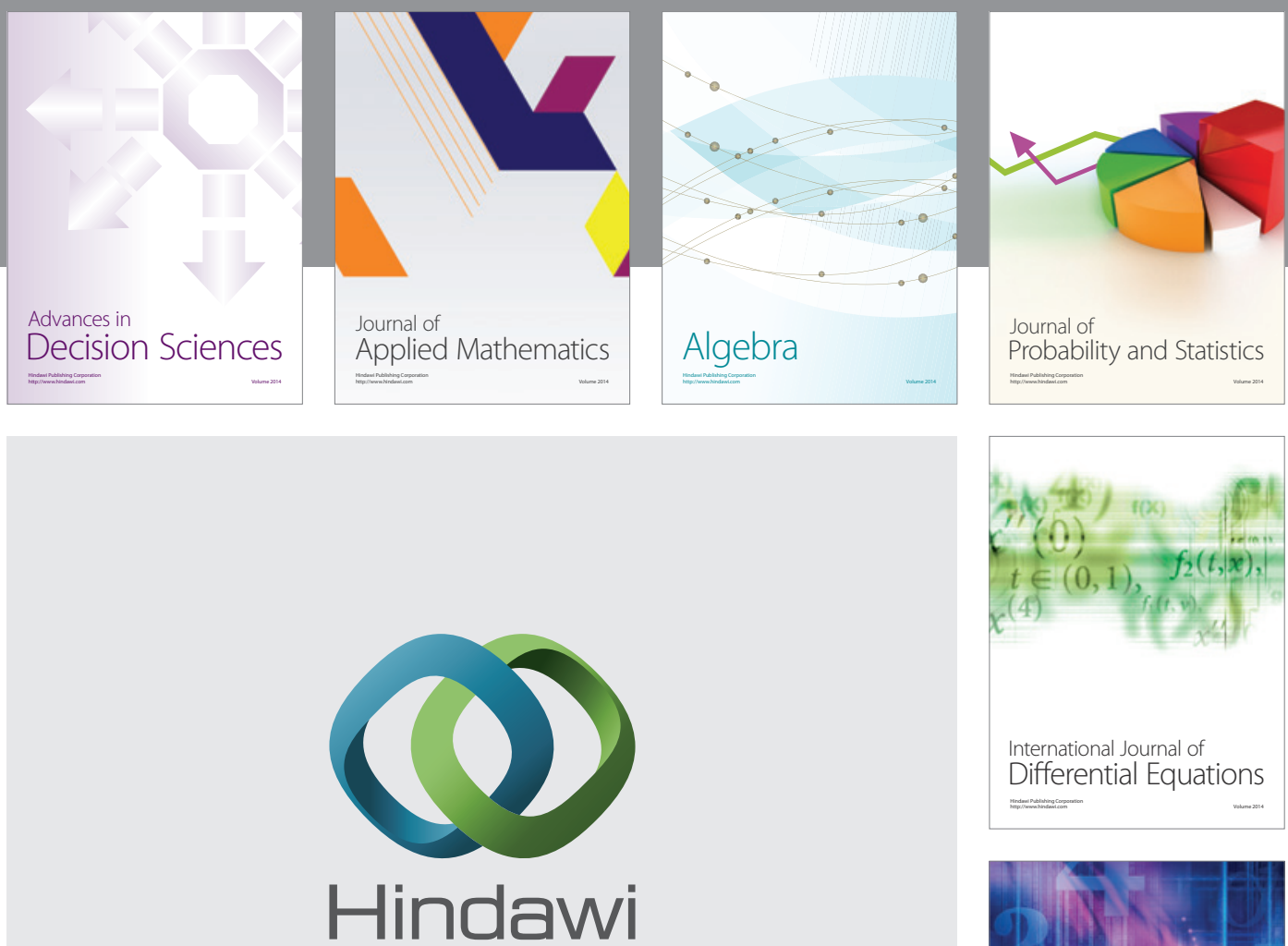

Submit your manuscripts at http://www.hindawi.com
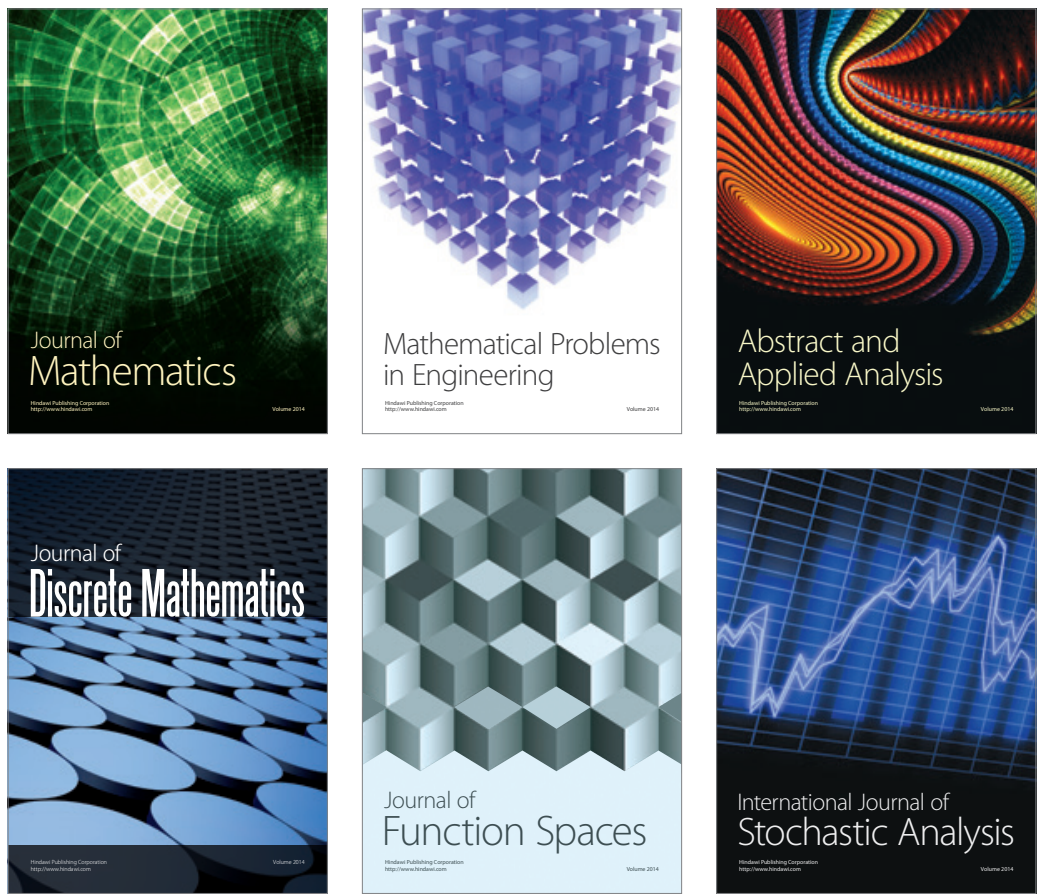

Journal of

Function Spaces

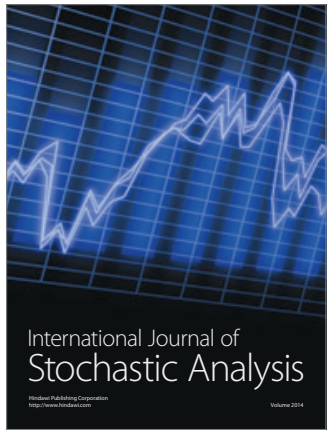

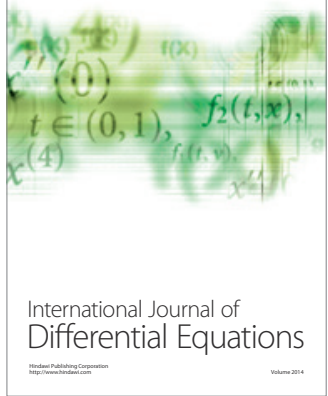
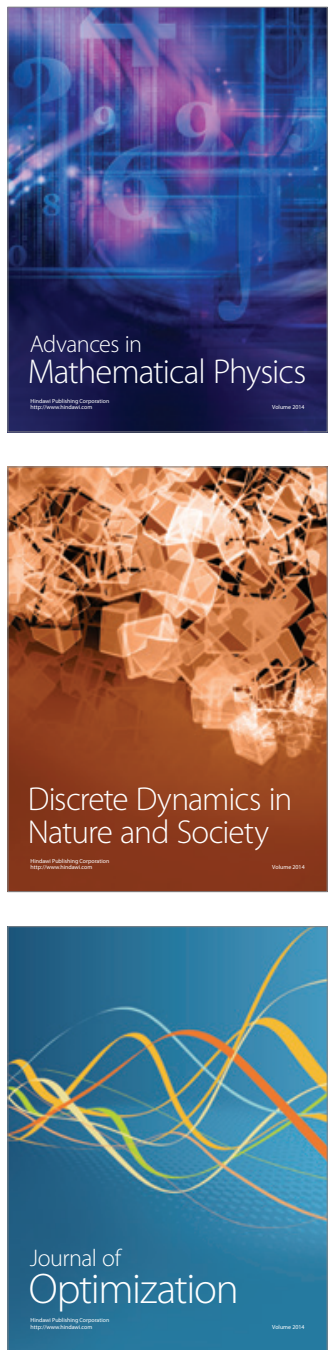